\title{
Toll-like receptor 4 blocker as potential therapy for acetaminophen-induced organ failure in mice
}

\author{
MOHAMED SALAMA ${ }^{1,2}$, MOHAMED ELGAMAL $^{1}$, AZZA ABDELAZIZ $^{3}$, MOATAZ ELLITHY $^{4}$, \\ DINA MAGDY ${ }^{4}$, LINA ALI ${ }^{4}$, EMAD FEKRY $^{2}$, ZINAB MOHSEN $^{1}$, MARIAM MOSTAFA $^{4}$, \\ HODA ELGAMAL $^{1}$, HUSSEIN SHEASHAA ${ }^{5}$ and MOHAMED SOBH ${ }^{1,5}$ \\ ${ }^{1}$ Medical Experimental Research Center; ${ }^{2}$ Toxicology Department; ${ }^{3}$ Mansoura-Manchester Programme; \\ ${ }^{4}$ Pathology Department; ${ }^{5}$ Urology and Nephrology Center, Faculty of Medicine, Mansoura University, Mansoura 36511, Egypt
}

Received July 24, 2014; Accepted April 7, 2015

DOI: $10.3892 /$ etm.2015.2442

\begin{abstract}
Acetaminophen (APAP, 4-hydroxyacetanilide) is the most common cause of acute liver failure in the United States. In addition to exhibiting hepatotoxicity, APAP exerts a nephrotoxic effect may be independent of the induced liver damage. Toll-like receptors (TLRs) have been suggested as a potential class of novel therapeutic targets. The aim of the present study was to investigate the potential of the TLR-4 blocker TAK-242 in the prevention of APAP-induced hepato-renal failure. Four groups of C57BL mice were studied: Vehicle-treated/control (VEH), APAP-treated (APAP), $\mathrm{N}$-acetyl cysteine (NAC)-pretreated plus APAP (APAP + NAC) and TAK-242-pretreated plus APAP (APAP + TAK) groups. Mice were clinically assessed then perfused $4 \mathrm{~h}$ later. Liver and kidney tissues were collected and examined histologically using basic hematoxylin and eosin staining to detect signs of necrosis and inflammation. Plasma samples were collected to measure the levels of alanine transaminase, aspartate transaminase and serum creatinine. In addition, liver and kidney tissues were assayed to determine the levels of reduced glutathione. The results of the present study indicate the potential role of TLR-4 in APAP-induced organ toxicity. In the APAP + TAK and APAP + NAC groups, histopathological examination indicated that pretreatment with TAK-242 or NAC afforded protection against APAP-induced injury. However, this protective effect was more clinically evident in the APAP + TAK group compared with the APAP + NAC group. The various biochemical parameters (serum enzymes and reduced
\end{abstract}

Correspondence to: Dr Mohamed Salama, Medical Experimental Research Center, Faculty of Medicine, Mansoura University, El Gomhourya Street, Mansoura 36511, Egypt

E-mail: toxicsalama@hotmail.com

Abbreviations: APAP, acetaminophen; NAC, N-acetyl cysteine; TLR-4, toll-like receptor 4; GSH, reduced glutathione

Key words: acetaminophen, toll-like receptor 4, hepatotoxicity, nephrotoxicity glutathione) revealed no significant protection in either of the pretreated groups. Therefore, the present study indicated that the TLR-4 blocker had protective effects against acute APAP toxicity in liver and kidney tissues. These effects were identified clinically, histologically and biochemically. Furthermore, the TLR-4 blocker TAK-242 exhibited antioxidant properties in addition to anti-inflammatory effects.

\section{Introduction}

Drug-induced liver injury (DILI) has been associated with $\sim 1,000$ types of drug (1) and is the most common reason for regulatory actions concerning drugs. DILI accounts for over half of the cases of acute liver failure, with acetaminophen (APAP, 4-hydroxyacetanilide) being the principal offending drug (2).

APAP is a widely used over-the-counter agent that exhibits antipyretic and analgesic activity. The hospitalization rate due to accidental and intentional APAP overdose has been estimated to be $>26,000$ cases per year in the USA (3). APAP-induced hepatic damage has been extensively investigated in mice as hepatic centrilobular necrosis occurs within hours following APAP administration (4,5). In addition to hepatotoxicity, APAP exerts a nephrotoxic effect that may be mechanistically independent of the liver damage it induces. In cases of nephrotoxicity, tubular cell loss is the characteristic feature of acute and chronic renal failure (6). Phase I metabolism of APAP, which is predominantly mediated by CYP2E1, produces toxic metabolites (7).

Protein binding is the critical initiating event underlying the cell death observed during APAP-induced liver injury. The subsequent results of this binding process include mitochondrial dysfunction, and oxidative stress and injury (8). Hence, the detection of reduced glutathione (GSH) serves as a useful marker of the injury cycle as it indicates the degree of oxidative stress 4induced. Although previous studies have suggested that binding to mitochondrial proteins is a key process in liver injury, an improved mechanistic understanding is required as other factors may also be involved (9). These include immunomodulators, for example, the toll-like receptors (TLRs).

TLRs are a family of transmembrane proteins that represent the major pattern recognition receptors (PRRs). TLR-2 
and TLR-4 are extracellular TLRs with a wide range of potential endogenous ligands, including heat shock proteins, high mobility group box 1 and breakdown products of fibronectin, heparin sulfate and hyaluronic acid (10). Binding of these endoligands to TLR-2 and TLR-4 leads to the stimulation of adaptor proteins, such as MYD88, TRIF, TRAF and NF- $\kappa \mathrm{B}$, followed by increased cytokine and chemokine production (11). The portal vein drains blood from the gastrointestinal tract and supplies $75-80 \%$ of the blood supply to the liver. Although a constant inflow of gut-derived microbes to the liver occurs, hepatic TLRs are not constantly activated. This high threshold for the activation of liver TLRs is known as 'tolerance'. Tolerance is associated with the low-level expression of TLRs and signaling molecules, such as MD2 and MYD88 (12), in addition to the upregulation of interleukin-1 receptor-associated kinase-M (13). Therapeutic manipulation of the hepatic TLR system may be key to the development of novel treatments for the management and treatment of chronic inflammatory liver diseases. Various animal models (14) and phase III clinical trials (15) involving the use of small molecules, such as lipid A and TAK-242 (a TLR-4 blocker/antagonist) have indicated the beneficial effects of these agents in the management of septic patients. Agents such as these may be of benefit in the management of various liver diseases. Furthermore, Xu et al (16) demonstrated that TLR-2 and TLR-4 are major receptors for extracellular histone-mediated sterile inflammation, tissue injury and death in a mouse model of APAP. These results support the use of TLRs as therapeutic targets. Therefore, the present study aimed to evaluate the role of anti-TLR-4 agents as a potential therapy for APAP-induced organ injury, which may act through immunomodulation and the mitigation of APAP-induced inflammatory processes. Furthermore, the TLR-4 antagonist treatment was compared with the conventional APAP therapy (N-acetyl cysteine, NAC) that reduces the oxidative stress induced by APAP toxicity.

\section{Materials and methods}

Study design. The efficiency of TAK-242 (Invitrogen Life Technologies, Carlsbad, CA, USA), a TLR-4 blocker, in the treatment of APAP-induced injury was evaluated by histological and biochemical analysis in a mouse model. Mice treated with TAK-242 exhibit downregulation of TLR-4 in liver and kidney tissue. The model of APAP toxicity used in the present study has been verified in previous studies $(4,5)$, which indicated that centrilobular liver damage was induced within a 4-h period post-treatment with APAP. This was verified again in the Medical Experimental Research Center (MERC) of Mansoura University (Mansoura, Egypt) prior to proceeding with this study. Reagents were purchased from Sigma-Aldrich, St. Louis, MO, USA, unless otherwise stated.

Animals. The present study was approved by the ethics committee of Mansoura University. A total of 40 male, 5-week-old C57BL/6 mice (Animal House, MERC, Mansoura University), weighing $16-20 \mathrm{~g}$, were maintained at $21-23^{\circ} \mathrm{C}$, with a humidity of $40-55 \%$ and $12 \mathrm{~h}$ light cycle (lights on 06:00-18:00). Mice were acclimatized for a 7-day period prior to the initiation of any procedures. Mice were fasted overnight, but had free access to water prior to the experiments.
Mice were allocated at random into the following groups ( $n=10$ per group): Vehicle-treated/control(VEH); APAP-treated (APAP); NAC-pretreated plus APAP (APAP + NAC); and TAK-242-pretreated plus APAP (APAP + TAK) groups. Mice were fasted as described above. Mice were injected intraperitoneally (ip) with $400 \mathrm{mg} / \mathrm{kg}$ APAP (26 mg/ml in water) or water (VEH) at 10:00 a.m. At $1 \mathrm{~h}$ prior to APAP or VEH injection (09:00 a.m.), mice were injected ip with $1.25 \mathrm{mmol} /$ $\mathrm{kg}$ NAC (204 mg/kg, $40 \mathrm{mg} / \mathrm{ml} \mathrm{pH} 7$ in water) or $1 \mathrm{mg} / \mathrm{kg}$ TAK-242. Plasma, and liver and kidney tissues were collected $4 \mathrm{~h}$ after APAP administration.

Animal assessment. Mice were examined for clinical manifestations of liver failure, such as disturbed sensorium, by an experienced researcher. Furthermore, following sacrifice by transcardial perfusion, the sizes and weights of the mouse livers were compared among groups.

Serum enzyme assays. Plasma samples were collected and stored at $4^{\circ} \mathrm{C}$ until required for the measurement of the levels of alanine transaminase (ALT), alanine aspartate (AST) and creatinine (ALT/AST activity kit, Sigma; creatinine assay kit, Erba Mannheim, Mannheim, Germany). The serum enzyme assay (Slim+ spectrophotometer, SEAC, Florence, Italy) was conducted to ensure the development of liver and kidney damage in all study groups.

Reduced glutathione (GSH) determination. Tissue samples (200 mg) were homogenized in $500 \mu \mathrm{l}$ sulfosalicylic acid $(0.5 \%)$ and adjusted to a volume of $1 \mathrm{ml}$. Total GSH was determined using GSH reductase and NADPH-coupled reaction, with 5,5'-dithiobis(2-nitrobenzoic acid), as previously described (17). Values are expressed as nmol/g tissue.

Histology. Segments of liver and kidney tissue were fixed in $15 \mathrm{ml}$ neutral buffered formalin solution. Tissue samples were embedded in Paraplast and processed as described previously (5). The tissue samples were sectioned to $\sim 4-\mu \mathrm{m}$ thickness and stained with hematoxylin and eosin (H\&E). The tissues were subsequently examined under a light microscope (CX31RTSF; Olympus, Tokyo, Japan).

Statistical analysis. Data are presented as the mean \pm standard deviation. Two groups of data were analyzed by Student's t-test. Three groups of data were analyzed by analysis of variance with a Tukey post hoc test. For all tests, $\mathrm{P}<0.05$ was considered to indicate a statistically significant difference.

\section{Results}

Animal assessment. Animals treated with APAP exhibited disturbed sensorium and hepatomegaly. By contrast, mice treated with NAC or TAK-242 exhibited improvements of these symptoms, which were more marked in the APAP + TAK group. The APAP + TAK group mice displayed improved normalization of liver size and weight compared with the APAP and APAP + NAC groups. Although NAC treatment resulted in a reduction in liver size, the APAP + NAC group mice exhibited significant hepatomegaly compared with the APAP + TAK and VEH group mice. 
Table I. Serum enzymes assay in different treatment groups.

\begin{tabular}{lccc}
\hline Group & ALT (IU/l) & AST (IU/l) & Creatinine $(\mu$ mol/l) \\
\hline VEH & $83.02 \pm 12.01$ & $91.11 \pm 11.20$ & $0.73 \pm 0.07$ \\
APAP & $800.10 \pm 27.11^{\mathrm{a}}$ & $1320.41 \pm 32.02^{\mathrm{a}}$ & $3.21 \pm 0.20^{\mathrm{a}}$ \\
APAP + NAC & $452.23 \pm 15.03^{\mathrm{a}, \mathrm{b}}$ & $789.72 \pm 22.10^{\mathrm{a}, \mathrm{b}}$ & $0.42 \pm 0.03^{\mathrm{b}}$ \\
APAP + TAK & $588.33 \pm 23.02^{\mathrm{a}, \mathrm{b}}$ & $757.32 \pm 33.13^{\mathrm{a}, \mathrm{b}}$ & $0.42 \pm 0.04^{\mathrm{a}, \mathrm{b}}$ \\
\hline
\end{tabular}

Data presented are mean \pm standard deviation. ${ }^{\mathrm{a}} \mathrm{P}<0.05$ vs. the VEH group. ${ }^{\mathrm{b}} \mathrm{P}<0.05$ vs. the APAP group. ALT, alanine transaminase; AST, aspartate transaminase; VEH, vehicle; APAP, acetaminophen; NAC, N-acetyl cysteine; TAK, TAK-242.

Table II. Levels of the oxidative stress marker GSH in the liver tissues of different treatment groups.

\begin{tabular}{lc}
\hline Group & GSH $(\mathrm{nmol} / \mathrm{g})$ \\
\hline VEH & $38.91 \pm 5.02$ \\
APAP & $1.25 \pm 0.04^{\mathrm{a}}$ \\
APAP + NAC & $12.26 \pm 0.27^{\mathrm{a}, \mathrm{b}}$ \\
APAP + TAK & $12.85 \pm 0.31^{\mathrm{a}, \mathrm{b}}$ \\
\hline
\end{tabular}

Data presented are mean \pm standard deviation. ${ }^{\mathrm{a}} \mathrm{P}<0.05$ vs. the $\mathrm{VEH}$ group. ${ }^{\mathrm{b}} \mathrm{P}<0.05$ vs. the APAP group. GSH, reduced glutathione; VEH, vehicle; APAP, acetaminophen; NAC, N-acetyl cysteine; TAK, TAK-242.
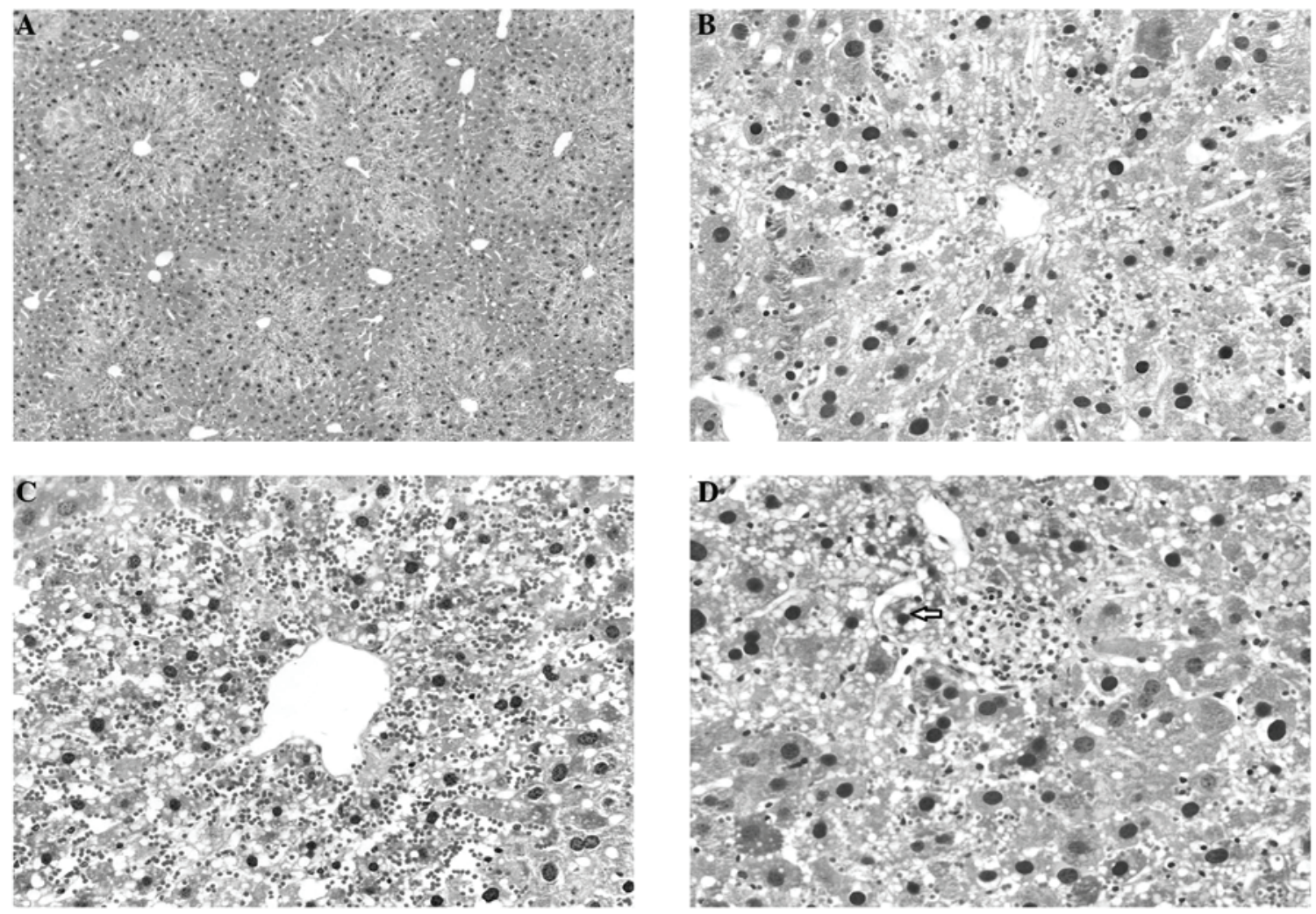

Figure 1. Liver tissues from the acetaminophen (APAP) group, presenting with liver centrilobular hemorrhage, degeneration and necroinflammation (multiple foci of focal lytic necrosis with replacement by inflammatory cells). (A) Centrilobular degeneration, necrosis and hemorrhage (H\&E staining, magnification, x100). (B and C) pericentral degeneration and hemorrhage and (D) pericentral degeneration, necroinflammation (arrow) and hemorrhage in APAP (H\&E staining; magnification, $\mathrm{x} 400)$. H\&E, hematoxylin and eosin. 

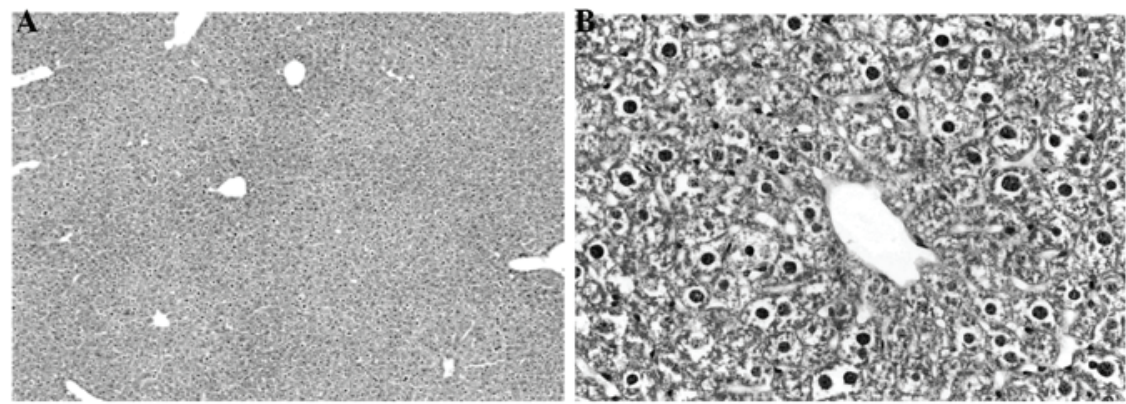

Figure 2. Histopathological analysis of liver tissue from the $\mathrm{N}$-acetyl cysteine treated group. (A) Exhibits mild change (H\&E staining; magnification, x100). (B) Mild degenerative changes, no hemorrhage or necroinflammation (H\&E staining; magnification, $\mathrm{x} 400)$. H\&E, hematoxylin and eosin.
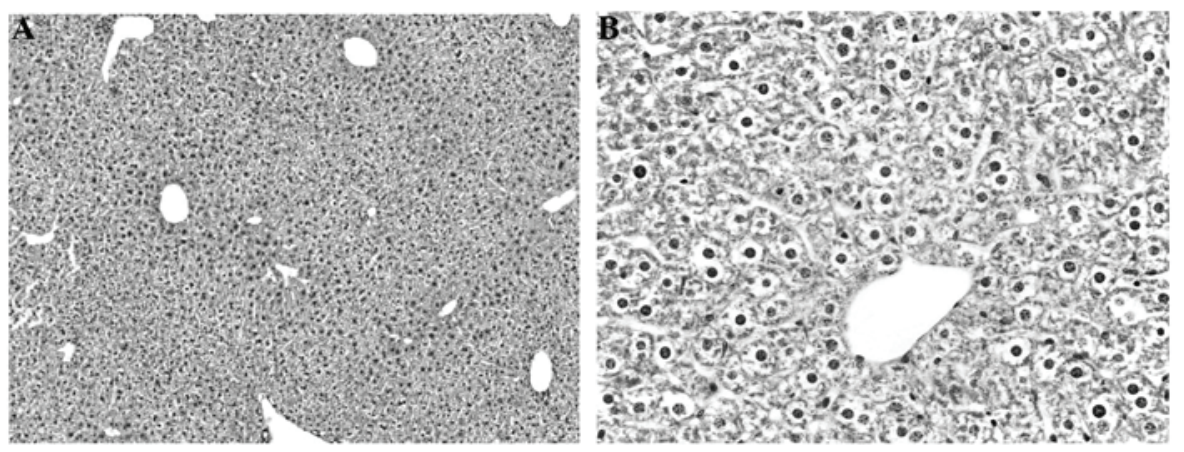

Figure 3. Histopathological findings of liver tissue from the TAK-242 treated group. (A) Mild change (H\&E staining; magnification, x100). (B) Mild degenerative changes, no hemorrhage and no necroinflammation (H\&E staining; magnification, x400). H\&E, hematoxylin and eosin.
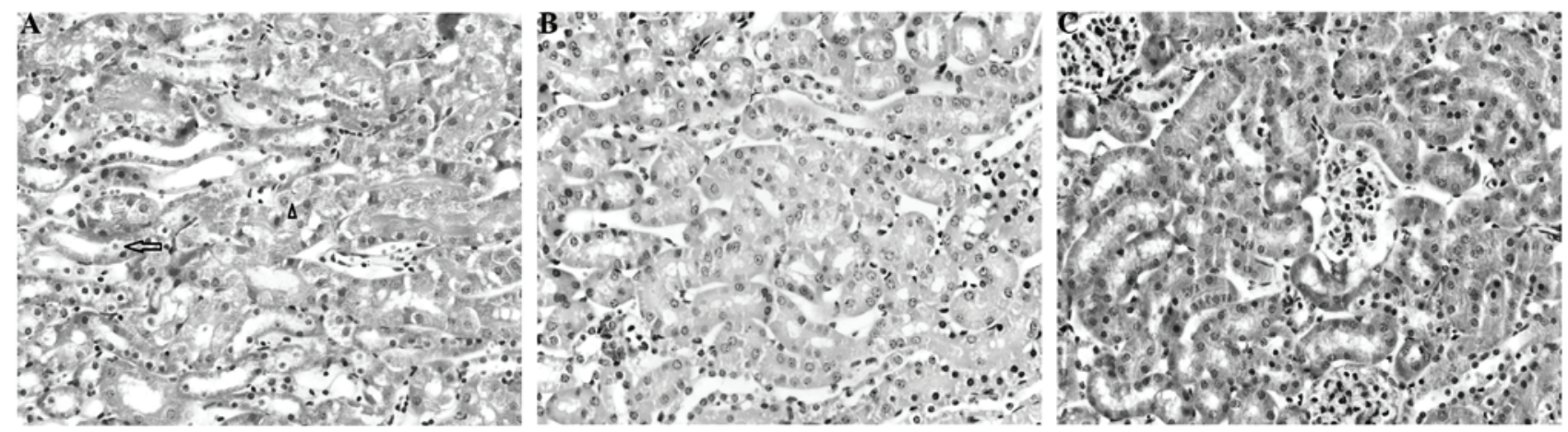

Figure 4. Histopathological changes in kidney tissue from the (A) acetaminophen (APAP), (B) APAP + N-acetyl cysteine and (C) APAP + TAK-242 treated groups. (A) Tubular cell necrosis (arrow) and apoptosis (arrow head); (B and C) no evidence of tubular cell necrosis or apoptosis (hematoxylin \& eosin staining, magnification, $\mathrm{x} 400)$.

Serum enzyme assays. The results of ALT, AST and creatinine assays for all groups are presented in Table I. Animals treated with APAP exhibited a significant increase in the serum levels of all three enzymes compared with those in the control group. By contrast, the APAP + NAC and APAP + TAK groups displayed normalized serum levels of creatinine, which were comparable to the level in the VEH group and significantly reduced compared with that in the APAP group. Furthermore, the APAP + NAC and APAP + TAK groups presented with reduced levels of ALT and AST compared with those in the APAP group. However, these levels remained increased in comparison with those in the VEH group.
GSH levels. Tables II and III display the levels of GSH in the liver and kidney tissues, respectively, of all study groups. GSH levels were higher in the VEH group mice than in the other groups. APAP treatment led to a significant reduction in GSH levels in the liver and kidney tissues. The APAP + NAC and APAP + TAK groups exhibited significant elevations in GSH levels in liver and kidneys compared with those in the APAP group; however, their levels remained reduced in comparison with those in the VEH group mice.

Histology. Figs. 1-4 display the results of H\&E staining of liver and kidney tissues. The mice in the APAP group exhibited liver 
centrilobular hemorrhage, degeneration and necroinflammation, with multiple foci of focal lytic necrosis with replacement by inflammatory cells (Fig. 1). The livers of the mice in the APAP + NAC and APAP + TAK groups exhibited mild degenerative changes, with no hemorrhage or necroinflammation (Figs. 2 and 3). Kidneys from the mice in the APAP group presented with focal tubular cell detachment, necrosis and apoptosis (Fig. 4A). However, the kidneys of the mice in the APAP + NAC and APAP + TAK groups displayed no evidence of tubular cell necrosis or apoptosis (Fig. 4B and C).

\section{Discussion}

In the present study, the role of TLR-4 in APAP-induced organ failure was investigated. This was conducted by blocking TLR-4 using TAK-242 prior to treating the mice with a toxic dose of APAP. Liver and kidney tissues were subsequently harvested and analyzed using $\mathrm{H} \& \mathrm{E}$ histopathology. The results indicated that blocking TLR-4 significantly protected the liver and kidney tissues against APAP toxicity. This approach may provide an alternative to the conventional NAC therapy for APAP, which exhibits a number of limitations. First, NAC produces certain side-effects (intractable vomiting, allergic reaction) in clinical practice (18). Furthermore, NAC therapy requires the administration of high doses of NAC over an extended treatment period (5). Blocking TLR-4 with the administration of a single dose may provide a more reliable alternative to the prolonged NAC protocol. The successful mitigation of APAP-induced hepato-renal toxicity by the administration of a TLR-4 antagonist in the present study supports the results of the study by Xu et al (16), which indicated a potential pathogenic role of TLR-4 in APAP toxicity. The results of the present study are consistent with the previous observations of Shah et al (19), which demonstrated the beneficial effects of TLR-4 blockade in an APAP toxicity model.

It is notable that the protective effects of blocking TLR-4 were observed in both liver and kidney tissues. This result underlines the crucial function of TLRs in various organs, and their role in systemic conditions, such as septicemia and toxicity (16). Furthermore, TLR-4 has been previously demonstrated to serve a key function in organ crosstalk (20). Thus, TLR-4 may protect tissues against APAP-induced nephrotoxicity that occurs directly, for example via toxic metabolites attacking renal targets, or indirectly through hepato-renal crosstalk.

The effect of TLR-4 blockade on oxidative stress was also evaluated in the present study, as it is well established that oxidative stress is involved in the pathogenesis of APAP-induced toxicity (21). Notably, blocking TLR-4 mitigated the effects of APAP-induced oxidative stress. The association between oxidative stress and inflammation is readily understood. Conditions that promote significant oxidative stress may precipitate cellular death and extracellular matrix (ECM) breakdown. Necrotic cells and damaged ECM in turn release various intracellular and extracellular molecules, which function as 'alarmins', triggering inflammatory cascades following recognition by PRRs (22). Furthermore, oxidative stress conditions may induce various modifications within lipids and proteins, generating so-called oxidation-specific epitopes. These function as potent damage-associated molecular patterns, and are able to trigger innate immune responses by binding to multiple
PRRs (23). Hence, previous studies support the hypothesis that antioxidant therapy may inhibit inflammatory processes by preventing the initiation of this cycle. However, in the present study, the reverse was observed; as the blocking of a proinflammatory agent, i.e. TLR-4, led to a reduction in oxidative stress. Similar results were obtained in a study of intestinal epithelial cells conducted by Latorre et al (24). Latorre et al observed that TLR-2, -3 and -4 activation may induce pro-oxidant effects. As a result, it was hypothesized that TLRs may possess pro-oxidant properties in addition to their inherent innate immunity properties. Another study of the association between TLR-4 and oxidative stress (25) indicated that the pro-oxidative properties of these molecules are not associated with their innate immunity modulatory effects. However, the mechanisms underlying these properties remain unspecified and require further evaluation.

In conclusion, the present study demonstrated the protective effects of the TLR-4 blocker TAK-242 against acute APAP toxicity in liver and kidney tissues. These effects were demonstrated clinically, histologically and biochemically. Furthermore, TAK-242 appeared to exert antioxidative effects in addition to its anticipated anti-inflammatory effects.

\section{Acknowledgements}

The present study was funded by a grant from the Medical Experimental Research Center of Mansoura University (no. 014-1).

\section{References}

1. Abboud G and Kaplowitz N: Drug-induced liver injury. Drug Saf 30: 277-294, 2007.

2. Tujios S and Fontana RJ: Mechanisms of drug-induced liver injury: From bedside to bench. Nat Rev Gastroenterol Hepatol 8: 202-211, 2011

3. Nourjah P, Ahmad SR, Karwoski C and Willy M: Estimates of acetaminophen (Paracetomal)-associated overdoses in the United States. Pharmacoepidemiol Drug Saf 15: 398-405, 2006.

4. Mitchell JR, Jollow DJ, Potter WZ, Gillette JR and Brodie BB: Acetaminophen-induced hepatic necrosis. IV. Protective role of glutathione. J Pharmacol Exp Ther 187: 211-217, 1973.

5. Terneus MV, Kiningham KK, Carpenter AB, Sullivan SB and Valentovic MA: Comparison of S-adenosyl-L-methionine and $\mathrm{N}$-acetylcysteine protective effects on acetaminophen hepatic toxicity. J Pharmacol Exp Ther 320: 99-107, 2007.

6. Cermik H, Taslipinar MY, Aydin I, et al: The relationship between $\mathrm{N}$-acetylcysteine, hyperbaric oxygen and inflammation in a rat model of acetaminophen-induced nephrotoxicity. Inflammation 36: 1145-1152, 2013.

7. McGill MR and Jaeschke H: Metabolism and disposition of acetaminophen: Recent advances in relation to hepatotoxicity and diagnosis. Pharm Res 30: 2174-2187, 2013

8. Jaeschke H, McGill MR and Ramachandran A: Oxidant stress, mitochondria and cell death mechanisms in drug-induced liver injury: Lessons learned from acetaminophen hepatotoxicity. Drug Metab Rev 44: 88-106, 2012.

9. Jaeschke $H$ and Bajt ML: Intracellular signaling mechanisms of acetaminophen-induced liver cell death. Toxicol Sci 89: 31-41, 2006.

10. Arslan F, Keogh B, McGuirk P and Parker AE: TLR2 and TLR4 in ischemia reperfusion injury. Mediators Inflamm 2010: 704202, 2010.

11. Zager RA, Johnson AC, Lund S and Randolph-Habecker J: Toll like receptor (TLR4) shedding and depletion: acute proximal tubular cell responses to hypoxic and toxic injury. Am J Physiol. Renal Physiol 292: F304-F312, 2007.

12. Hayashi F, Smith KD, Ozinsky A, et al: The innate immune response to bacterial flagellin is mediated by Toll-like receptor 5 . Nature 410: 1099-1103, 2001. 
13. Kobayashi K, Hernandez LD, Galán JE, Janeway CA Jr, Medzhitov R and Flavell RA: IRAK-M is a negative regulator of Toll-like receptor signaling. Cell 110: 191-202, 2002.

14. Daubeuf B, Mathison J, Spiller S, et al: TLR4/MD-2 monoclonal antibody therapy affords protection in experimental models of septic shock. J Immunol 179: 6107-6114, 2007.

15. Kanzler H, Barrat FJ, Hessel EM and Coffman RL: Therapeutic targeting of innate immunity with Toll-like receptor agonists and antagonists. Nat Med 13: 552-559, 2007

16. Xu J, Zhang X, Monestier M, Esmon NL and Esmon CT: Extracellular histones are mediators of death through TLR2 and TLR4 in mouse fatal liver injury. J Immunol 187: 2626-2631, 2011

17. Valentovic M, Terneus M, Harmon RC and Carpenter AB: S-Adenosylmethionine (SAMe) attenuates acetaminophen hepatotoxicity in C57BL/6 mice. Toxicol Lett 154: 165-174, 2004.

18. Kao LW, Kirk MA, Furbee RB, Mehta NH, Skinner JR and Brizendine EJ: What is the rate of adverse events after oral $\mathrm{N}$-acetylcysteine administered by the intravenous route to patients with suspected acetaminophen poisoning? Ann. Emerg Med 42: 741-750, 2003

19. Shah N, Montes de Oca M, Jover-Cobos M, et al: Role of toll-like receptor 4 in mediating multiorgan dysfunction in mice with acetaminophen induced acute liver failure. Liver Transpl 19: 751-761, 2013.
20. Salama M, Farrag SM, Abulasrar SA, et al: Up-regulation of TLR-4 in the brain after ischemic kidney-induced encephalopathy in the rat. CNS Neurol Disord Drug Targets 12: 583-586, 2013.

21. Hartley DP, Kolaja KL, Reichard J and Petersen DR 4-Hydroxynonenal and malondialdehyde hepatic protein adducts in rats treated with carbon tetrachloride: Immunochemical detection and lobular localization. Toxicol Appl Pharmacol 161: 23-33, 1999.

22. Chan JK, Roth J, Oppenheim JJ, et al: Alarmins: Awaiting a clinical response. J Clin Invest 122: 2711-2719, 2012.

23. Lugrin J, Rosenblatt-Velin N, Parapanov R and Liaudet L: The role of oxidative stress during inflammatory processes. Biol Chem 395: 203-230, 2014.

24. Latorre E, Mendoza C, Layunta E, Alcalde AI and Mesonero JE: TLR2, TLR 3 and TLR4 activation specifically alters the oxidative status of intestinal epithelial cells. Cell Stress Chaperones 19: 289-293, 2014

25. Pierre N, Deldicque L, Barbé C, Naslain D, Cani PD and Francaux M: Toll-like receptor 4 knockout mice are protected against endoplasmic reticulum stress induced by a high-fat diet PLoS One 8: e65061, 2013. 